\section{Countrywide demonstration of adult protection derived from infant immunisation}

\author{
Carlos G Grijalva ${ }^{1,2}$
}

Humans, especially young children, are considered the only reservoir of Streptococcus pneumoniae. Pneumococcal nasopharyngeal colonisation is very common and usually asymptomatic, but it also represents the primordial step in the development of pneumococcal diseases. ${ }^{1}$ Nasopharyngeal colonisation is very prevalent among young children but appears to be much less common among adults. Yet, adults, especially older adults, are disproportionally affected by pneumococcal diseases. Close interaction with young children is a recognised source of exposure to pneumococcus among adults, and previous studies have documented the associations with colonisation and also disease among exposed adults. ${ }^{2-4}$

The chain of transmission of pneumococcal colonisation and its connection with disease has been drastically transformed with the introduction of the pneumococcal conjugate vaccines. Unlike non-conjugated polysaccharide vaccines, pneumococcal conjugate vaccines elicit a strong immune response in vaccinated young children and provide protection against colonisation with serotypes included in the vaccine. This leads not only to protection of vaccinated children, but by targeting the main reservoir of transmission, vaccination also prevents transmission of pneumococcus to others in contact with vaccinated children. Indeed, widespread vaccination of infants with conjugate vaccines has led first to reductions and ultimately to virtual elimination of the circulation of serotypes included in the vaccines. ${ }^{5-8}$

Routine vaccination of infants with pneumococcal conjugate vaccines has led to profound changes in the ecology of $S$. pneumoniae and the distribution of related infections. As the circulation of vaccine

\footnotetext{
${ }^{1}$ Division of Pharmacoepidemiology, Department of Health Policy, Vanderbilt University Medical Center, Nashville, Tennessee, USA

${ }^{2}$ Health Services Research and Development Center, Veterans Health Administration-Tennessee Valley Healthcare System Geriatric Research Education Clinical Center (GRECC), Nashville, Tennessee, USA
}

Correspondence to Dr Carlos G Grijalva, Division of Pharmacoepidemiology, Department of Health Policy, Vanderbilt University Medical Center, Nashville, TN 37232,USA; carlos.grijalva@vanderbilt.edu serotypes was reduced due to the powerful biological pressure of the vaccine, disease caused by those retreating vaccine serotypes has declined in both vaccinated and unvaccinated groups. The protection among unvaccinated groups or indirect protection effects were documented first in populations that adopted the pneumococcal conjugate vaccination programmes early, but with the close to global adoption of these vaccination programmes, there is now a growing body of evidence demonstrating the direct and indirect protection derived from vaccination of infants with pneumococcal conjugate vaccines. ${ }^{6-9}$

Pneumococcal diseases encompass a number of conditions, including invasive and non-invasive diseases. Invasive diseases are typically defined by isolation of the pneumococcus, which allows the characterisation (ie, serotyping) of the causal pathogen. Surveillance systems that monitor invasive pneumococcal diseases have used these highly specific outcomes to demonstrate that after introduction of pneumococcal conjugate vaccination programmes, the incidence of invasive disease due to vaccine serotypes among vaccinated and unvaccinated groups has declined. However, invasive pneumococcal diseases are rare, relative to other non-invasive pneumococcal diseases, such as pneumonia. Unlike invasive diseases, assessments of the vaccination impact on pneumonia typically lack detailed serotype information to enable more specific determinations or to directly monitor the magnitude of serotype replacement. ${ }^{10}$ Nonetheless, under the consideration that a substantial fraction of pneumonias is caused by the pneumococcus, previous studies have focused on hospitalisations for all-cause pneumonia to estimate potential changes following vaccine introduction. Although the direct protection of vaccinated children against pneumonia seems well established, several-but not all-studies of unvaccinated adults have documented reductions in pneumonia hospitalisations after implementation of routine vaccination of infants with pneumococcal conjugate vaccines. ${ }^{6} 11-20$

In Thorax, Omar Okasha and colleagues report the findings of a countrywide assessment of indirect protection of adults derived from the Finnish infant pneumococcal conjugate vaccination programme. ${ }^{21}$ Although most previous assessments have focused on a 7 or 13 -valent pneumococcal conjugate vaccine, this study evaluated a vaccination programme with a 10 -valent vaccine (PCV10). The national vaccination programme started in September 2010, and eligible children were vaccinated following a $2+1$ schedule, with no catch-up programme. The use of either pneumococcal polysaccharide or conjugate vaccine among adults during the study period was negligible. Using comprehensive national registry data from 2004 to 2015, and an ecologic study design, the authors modelled the national incidence of pneumonia hospitalisations in adults during the study period, to examine whether vaccination of infants led to indirect protection of unvaccinated adults. Before introduction of the programme, pneumonia hospitalisation rates were following an increasing trend, which was clearly reversed after introduction of the programme. Overall, there was a $15.4 \%$ reduction in the incidence of all-cause pneumonia hospitalisations among unvaccinated adults, when comparing the observed rates with the projected rates that would have been observed if no vaccination programme was implemented. At 4 years after introduction of the programme, the observed relative declines were more pronounced among young than among older adults. Nevertheless, the estimated absolute reductions among older adults were sizeable. The authors concluded that approximately 4 years after introduction of the vaccination programme, there has been a significant impact in the incidence of all-cause pneumonia hospitalisations among unvaccinated adults. Larger declines in the overall incidence of the more specific, but much less common, pneumococcal pneumonia were also demonstrated, but no significant changes in empyema were observed.

In the study, remarkable efforts were made to account for potential alternate explanations for the observed declines. Although the primary analysis focused on hospitalisations with a primary diagnosis of pneumonia, a secondary analysis identifying pneumonia in any diagnosis position yielded similar relative reductions as those from the main analysis. Other analyses assessed the potential influence of new pneumonia management guidelines, and showed that no changes occurred following guidelines release in 2008, and that those changes only started after the introduction of the pneumococcal 
conjugate vaccination programme. Similarly, separate examinations that were not restricted to pneumonia hospitalisations, including outpatient and emergency department visits, demonstrated reductions consistent with those observed in the inpatient setting alone. Investigators also examined all-cause hospitalisations, excluding pneumonia, as a control condition. This examination showed a continuous declining trend throughout the study period, with no evident interruptions that coincided temporally with the introduction of the vaccination programme. This observation indicates that the observed changes in pneumonia hospitalisations cannot be explained by general changes in hospitalisation patterns. Potential limitations of the study were also appropriately identified and discussed in this well-conducted thorough examination of indirect protection of unvaccinated adults.

It is important to note that the initial observations reported by Okasha and colleagues may represent just part of an evolving process. Although previous reports have documented indirect protection starting rapidly within a few years after vaccines introduction, this indirect protection is expected to continue expanding as vaccination programmes mature. A recent systematic review estimated that on average, a 90\% reduction in invasive pneumococcal disease through indirect protection would be attained after about 9 years of continued vaccination of infants. ${ }^{6}$ Therefore, follow-up studies would be useful to continue monitoring the impact of the Finnish vaccination programme beyond the initial 4 years after implementation period reported in the article.

This timely assessment also contributes to the growing body of evidence that supports an indirect protection of adults against pneumonia hospitalisations derived from vaccination of infants with pneumococcal conjugate vaccines. ${ }^{611-151920}$ Although there is an adult pneumococcal conjugate vaccine available, many countries are currently evaluating whether to recommend direct vaccination of adults or rely on indirect protection derived from the infant vaccination programme as demonstrated in this article. Those assessments need to consider the plausibility of the indirect protection in particular settings, and how fast the unvaccinated population would derive indirect protection. There are also different vaccination schedules available, and different costs related to those. ${ }^{78}$ Factoring the costs of the vaccination programmes is another important consideration for the ongoing discussions. Carefully conducted studies of indirect protection, like the one reported by Okasha and colleagues, provide important evidence to inform decisions about the protection of adults against pneumococcal diseases.

Funding This work was funded in part by the National Institutes of Health (R01AG043471).

Competing interests CGG has served as consultant for Pfizer and Merck. He has received research support from the National Institutes of Health, the Centers for Disease Control and Prevention, the Agency for Health Care Research and Quality, the Campbell Alliance, and the Food and Drug Administration.

Provenance and peer review Commissioned; externally peer reviewed.

(c) Article author(s) (or their employer(s) unless otherwise stated in the text of the article) 2018. All rights reserved. No commercial use is permitted unless otherwise expressly granted.

Check for updates

To cite Grijalva CG. Thorax 2018;73:208-209.

Published Online First 9 January 2018

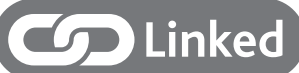

- http://dx.doi.org/10.1136/thoraxjnl-2017-210440

Thorax 2018;73:208-209

doi:10.1136/thoraxinl-2017-211099

\section{REFERENCES}

1 Käyhty H, Auranen K, Nohynek H, et al. Nasopharyngeal colonization: a target for pneumococcal vaccination. Expert Rev Vaccines 2006:5:651-67.

2 Walter ND, Taylor TH, Dowell SF, et al. Holiday spikes in pneumococcal disease among older adults. N Eng/ J Med 2009:361:2584-5.

3 Regev-Yochay G, Raz M, Dagan R, et al. Nasopharyngeal carriage of Streptococcus pneumoniae by adults and children in community and family settings. Clin Infect Dis 2004;38:632-9.

4 O'Brien KL, Dagan R. The potential indirect effect of conjugate pneumococcal vaccines. Vaccine 2003;21:1815-25.

5 Lee GM, Kleinman K, Pelton S, et al. Immunization, Antibiotic Use, and Pneumococcal Colonization Over a 15-Year Period. Pediatrics 2017;140:e20170001.

6 Shiri T, Datta S, Madan J, et al. Indirect effects of childhood pneumococcal conjugate vaccination on invasive pneumococcal disease: a systematic review and meta-analysis. Lancet Glob Health 2017:5:e51-e59.
7 Loo JD, Conklin L, Fleming-Dutra KE, et al. Systematic review of the indirect effect of pneumococcal conjugate vaccine dosing schedules on pneumococcal disease and colonization. Pediatr Infect Dis J 2014;33(Suppl 2):S161-S171.

8 Fleming-Dutra KE, Conklin L, Loo JD, et al. Systematic review of the effect of pneumococcal conjugate vaccine dosing schedules on vaccinetype nasopharyngeal carriage. Pediatr Infect Dis J 2014;33(Suppl 2):S152-S160.

9 Loo JD, Conklin L, Fleming-Dutra KE, et al. Systematic review of the effect of pneumococcal conjugate vaccine dosing schedules on prevention of pneumonia. Pediatr Infect Dis J 2014;33(Suppl 2):S140-S151.

10 Weinberger DM, Malley R, Lipsitch M. Serotype replacement in disease after pneumococcal vaccination. Lancet 2011:378:1962-73.

11 van Werkhoven $\mathrm{CH}$, Hollingsworth RC, Huijts SM, et al. Pneumococcal conjugate vaccine herd effects on noninvasive pneumococcal pneumonia in elderly. Vaccine 2016;34:3275-82.

12 Rodrigo C, Bewick T, Sheppard C, et al. Impact of infant 13-valent pneumococcal conjugate vaccine on serotypes in adult pneumonia. Eur Respir $\rfloor$ 2015;45:1632-41.

13 Grijalva CG, Nuorti JP, Arbogast PG, et al. Decline in pneumonia admissions after routine childhood immunisation with pneumococcal conjugate vaccine in the USA: a time-series analysis. Lancet 2007;369:1179-86.

14 Simonsen L, Taylor RJ, Schuck-Paim C, et al. Effect of 13-valent pneumococcal conjugate vaccine on admissions to hospital 2 years after its introduction in the USA: a time series analysis. Lancet Respir Med 2014;2:387-94

15 Griffin MR, Zhu Y, Moore MR, et al. U.S. hospitalizations for pneumonia after a decade of pneumococcal vaccination. $N$ Engl I Med 2013:369:155-63.

16 Bruhn CA, Hetterich S, Schuck-Paim C, et al. Estimating the population-level impact of vaccines using synthetic controls. Proc Natl Acad Sci U SA 2017;114:1524-9.

17 Menzies RI, Jardine A, McIntyre PB. Pneumonia in elderly australians: reduction in presumptive pneumococcal hospitalizations but no change in allcause pneumonia hospitalizations following 7-valent pneumococcal conjugate vaccination. Clin Infect Dis 2015;61:927-33.

18 Jardine A, Menzies RI, Mclntyre PB. Reduction in hospitalizations for pneumonia associated with the introduction of a pneumococcal conjugate vaccination schedule without a booster dose in Australia. Pediatr Infect Dis J 2010;29:607-12.

19 Luca DL, Kwong JC, Chu A, et al. Impact of pneumococcal vaccination on pneumonia hospitalizations and related costs in ontario: A population-based ecological study. Clin Infect Dis 2017.

20 Andrade AL, Afonso ET, Minamisava R, et al. Direct and indirect impact of 10-valent pneumococcal conjugate vaccine introduction on pneumonia hospitalizations and economic burden in all agegroups in Brazil: A time-series analysis. PLoS One 2017;12:e0184204

21 Okasha O, Rinta-Kokko H, Palmu AA, et al. Populationlevel impact of infant 10-valent pneumococcal conjugate vaccination on adult pneumonia hospitalisations in Finland. Thorax 2018;73:262-9. 